\title{
The Internet of Things to Support Urban Incident Responses
}

\author{
Rodrigo Santos $^{1}$, Javier Orozco ${ }^{1}$, Sergio F. Ochoa ${ }^{2}$, and Roc Meseguer ${ }^{3}$ \\ ${ }^{1}$ Department of Electrical Engineering and Computers, IIIE, UNS-CONICET, Argentina \\ ${ }^{2}$ Computer Science Department, Universidad de Chile, Chile \\ ${ }^{3}$ Computer Science Department, Universidad Politécnica de Cataluña, Spain
}

\begin{abstract}
Although the technological components required to implement Internet of Things (IoT) inspired solutions are already available, in most application scenarios it is not clear how to structure and combine them to reach a certain global behavior of a system. This paper proposes an architecture that helps design IoT-based systems that support the first responses during medium-size or large urban incidents. The main components of this architecture are characterized and the interactions between them are also specified. The usefulness of the proposed architecture is illustrated through its hypothetical use in a real urban emergency. The use of this proposal could be extended to other application areas such as security operatives and monitoring of patients at home.
\end{abstract}

Keywords: Internet of things, human-based wireless sensor networks, emergency response, urban emergencies, ambient intelligence, information sharing.

\section{Introduction}

Medium and large emergencies affecting urban areas (e.g. train derailments, fires affecting buildings, and accidental or intentional explosions) usually represent a challenging situation for first responders, due the lack of event early detection systems, mechanisms to perform a quick diagnose of the situation, and supporting information to make decisions in a distributed way. The complexity of the urban scenarios usually increases this challenge.

Immediately after an incident occurs, a 911 service receives the emergency calls. The operators receiving these calls has to determine the veracity of the emergency, and then perform a first diagnose of the incident (type, size and complexity), using the information provided by the people [12]. Based on such a diagnosis, the operators dispatch resources (mainly firefighters, police officers, and paramedics) to perform the first response process. The diagnosis and dispatching processes can take several minutes.

The response process involves resources transportation (e.g. fire trucks, ambulances, police vehicles, and first responders) that have to arrive quickly to the emergency place. The reaction time and the efficiency in the first response process are crucial to reduce the number of victims in an emergency [12], [14]. In [3] it is proved that reducing the first response time by 1 minute, correlates to a six percent difference 
in the number of lives saved in car crashes. These numbers are probably representative of other urban incidents.

The coordination of the emergency response activities represent a major challenge for first responders due to several reasons: (1) civilians usually go to the incident place to see what is happening, obstructing thus the resources transportation and response process, (2) services in the area tends to collapse (e.g. communication and transportation) or they are temporarily suspended for safety reasons (e.g. energy, gas and water), (3) there is little or no supporting information to make decisions and coordinate the efforts among the participants, and (4) the available radio channels used by first responders (e.g. firefighters, police officers, paramedic, and emergency managers) are not enough to coordinate the response activities. Therefore, the regular pattern in these situations is the improvisation of the first response process [10], [11].

This paper shows how regular sensing systems deployed in the affected areas can be used to help improve the efficiency and effectiveness of first response processes after medium-size or large urban incidents. The proposal is based on the Internet of Things (IoT) paradigm [4] and uses a Human-centric Wireless Sensor Network (HWSN) [13] to provide some intelligence to the response process. Particularly, an architecture that integrates information providers and consumers is described, indicating how to support the shared information flow among the participants. The use of the proposed architecture is illustrated analyzing a real first response process, which addressed a train crash happened at the Once train station, in Buenos Aires city (Argentina) in February $22^{\text {nd }}, 2012$. The article analyzes how the proposed architecture could have helped improve the reaction time and response activities after such an incident.

Next section presents and discusses the related work. Section 3 describes the proposed architecture. Section 4 exemplifies, using a real urban emergency situation, how the architecture could have contributed to reduce the incident impact. Section 5 presents the conclusions and the future work.

\section{Related Work}

The use of mobile devices, wireless sensor networks, and even the use of IoT to support first responses in urban incidents, has been addressed by several researchers. Concerning the incident detection, White et al. [16] proposed an automatic mechanism for traffic accident detection and notification using smart-phones. It delivers early alerts to particular emergency centers and thus accelerates the response process. Similarly, Liu et al. [8] use cyberphysical elements (called intelligent guards) deployed in the physical infrastructure to deliver early alarms when an extreme event affects a certain urban area.

Concerning the support for the first response process, Martin-Campillo et al. [9] propose a RFID-based solution to tag injured people, indicating their health condition before to deliver them to a hospital. Several researchers propose the use of mobile ad hoc networks, usually implemented using $\mathrm{Wi}-\mathrm{Fi}$, to provide communications support in disaster areas [1], [11], [15]. Ochoa and Santos [13] go a step forward and introduce the concept of Human-centric Wireless Sensor Networks (HWSN). They also show how HWSN-based solutions can be used to increase the information availability in the affected area. 
Zhang et al. [18] describes an IoT-inspired platform, which was designed to support emergency management systems. Although such a proposal is quite general, it allows seeing how the several components participating in the process can interact among them to capture or disseminate shared information.

Yang et al. [17] propose a modified "task-technology fit" approach to help understand how the IoT technology can enhance the urban first response activities. This approach does not indicate how to design IoT solutions to support these operations, but it allows us to realize the role of IoT technology in urban emergencies.

Although the previous proposals are interesting and contribute to improve the effectiveness of the first response process, none of them presents an architecture that indicates the type of components participating in the solution, the role played by each of them, and the way in which the shared information is captured and disseminated using these components. This article proposes an architecture inspired in the IoT paradigms, which uses a HWSN to determine components roles, and also the support required for information dissemination and fusion. Next section describes the proposed architecture, its main components and interactions among them.

\section{The IoT-Inspired Architecture}

Internet of Things infrastructures allow data and services integration among smart objects (e.g. mobile robots), sensing devices and human beings, using different but interoperable communication protocols [6]. Following this definition, Fig. 1 shows the architecture proposed for systems that support first responders after medium-size or large urban incidents. The architecture involves four layers that implement the separation of concerns: sensing, communication, information persistence and application (i.e. usage).

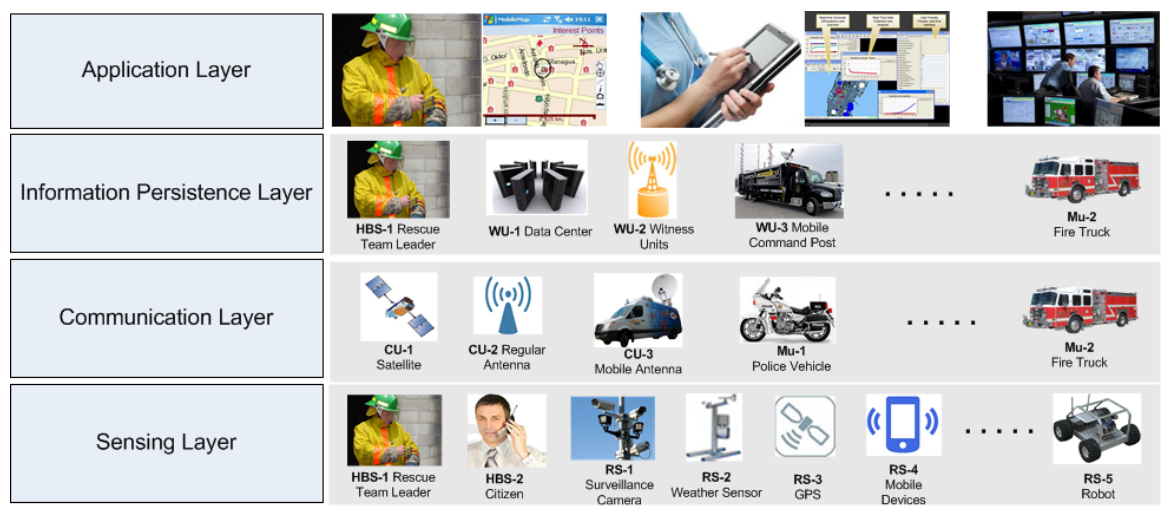

Fig. 1. Hierarchy of architectural components

The sensing layer is responsible to capture information from the field, which will be then used to support the decision making and coordination activities. Two types of components contribute to perform this activity: regular sensors (RS) and humanbased sensors (HBS). The first ones (e.g. weather and motion sensors, or 
video-camera) capture information from the environment and transmit it through a component of the communication layer. The HBS perform the same activity, however these sensors are people that also uses his senses to capture additional the information from the environment. Using such information, and eventually the data given by regular sensors, the HBS produce knowledge that represents the current value of a certain context variable (e.g. the emergency type or size). Although the HBS are not accurate, they represent our best choice when the observed variable is not measurable by a regular sensor.

The HBS uses a mobile device that allows him to share that knowledge with others and also to sense context variables (see the sensing layer in Fig. 1), e.g. the presence of other responders in the area by using an opportunistic network (oppnet). It is assumed that every component participating in this solution has a network interface that allows it to communicate with others through a digital network interface.

The communication layer is responsible to provide interaction capability to components participating in the first response process. Because there is not a universal network interface, this layer is implemented as a set of heterogeneous solutions, hopefully linked through communication bridges (Fig. 2a). Typically two types of communication solutions are used in these scenarios: infrastructure-based and ad hoc networks. The first one uses the regular communication infrastructure (i.e. satellites, cellular antennas and wired networks) and also mobile antennas to communicate the resources in the affected area, with remote components (e.g. emergency offices, data centers, remote experts, government agencies). We call communication units (CU) to these components that link resources inside the affected area with those that are outside the emergency place (see Fig. 1). Thus, these CUs allow the remote gathering and analysis of information (e.g. through Internet) that comes from sensors deployed in the emergency area (Fig. 2b).

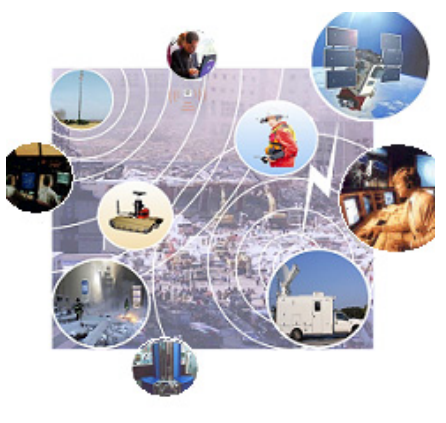

(a)

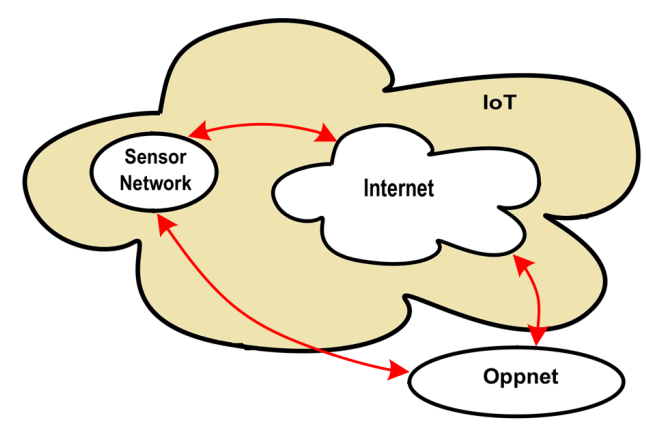

(b)

Fig. 2. Communication Infrastructure

Concerning the support based on ad hoc networks, its role is to provide and enhance the communication links in the field, increasing thus the information availability in that area and reducing the improvisation during the response process [10], [11], [13]. The use of opportunistic networks (oppnet) is highly recommended because they can work although the regular communication infrastructure is not available. An opp- 
net is a peer-to-peer application-oriented mesh, able to support ad hoc interactions among stationary and mobile units that are physically close; e.g. sensors, humanbased sensors and communication units deployed in the affected area. The oppnets are built in the application layer and they use a "store and forward" paradigm for transmitting messages [7]. The nodes participating in these networks can act as gateways bridging oppnets and regular Internet channels, allowing thus services integration and information exchange according to the IoT paradigm.

Provided that oppnets have quite short communication threshold, a special type of mobile node helps connect disjoint networks, allowing thus asynchronous communication among resources in the field. These mobile nodes, known as "mules" (Mu), are typically implemented through computing devices installed on police vehicles, fire trucks and ambulances (see the communication layer in Fig. 1).

The information persistence layer is responsible to store and share the supporting information, allowing participants to coordinate their activities and make better and on-time decisions. Several types of components can play this role, for instance the HBS, Mu and Witness Units (WU). The WU are buffers used on-demand by other nodes to store shared information. These WU also act as information gateway, particularly if they are accessible through Internet.

Finally the application layer is responsible to provide a direct and useful service to the end-users, e.g. first responders, incident commanders, emergency managers, government agents, hospital personnel and civilians (regular drivers). These applications make use of the services provided by the rest of the proposed architecture.

The role, behavior and services of every component type described in the architecture are clearly delimited. Therefore they can be formalized in a computable language, and then used with several purposes; for instance to evaluate vulnerability of a certain urban area, design response plans during a preparedness or response phase, and also to learn after an incident. Several emergency response agencies can take advantage of it to design not only their own response processes, but also the coordination of activities with other agencies, which is a recurrent limitation reported by the researchers after every medium-size and large incident.

The technology required to implement emergency response solutions adhering to the proposed architecture is available, and part of it is already deployed in many public spaces; i.e. Wi-Fi and GSM antennas, mobile devices with several communication capabilities, surveillance cameras, public speakers and displays, remotely controlled semaphores, traffic sensors, weather sensors, and ad hoc communication and positioning services. Only considering these regular components it is possible to design solutions to make more effective the response process to an urban incident.

\section{$4 \quad$ Application Example}

In order to exemplify the usefulness of this proposal, we will analyze some aspects of the response process conducted by first response task forces on February 22nd, 2012, after a train crash at a central station in Buenos Aires, Argentina. After 8:30 AM a train crashed with the end of line at the Once station. The accident leave fifty two dead people and over seven hundred injured. 
The Once station is the third one in importance in Buenos Aires. It is the head line for a very large rail network that goes to the west of the city. Only $3 \mathrm{~km}$ away from the city center, this station is a hub for buses, subway and other train lines. Every day one million people go through this hub for transportation connections (train-bussubway) or as their final station.

When the accident took place, the diagnose process involved various minutes due the complexity of the physical scenario, and it began when the first firefighters company arrived to the place. Although the accident was recorded by various surveillance cameras (i.e. a regular sensor), neither automatic alarms were delivered nor video records were shared with the emergency centers that could have helped to reduce the reaction time. Analyzing the accident video records it is possible to estimate the size and type of the emergency in approximately one minute. Therefore, sharing this video with the proper emergency center could have allowed triggering a quick response and saving more lives.

Once known the first diagnose and according to the protocol, the emergency center (i.e. the end-user) performed a formal dispatch of emergency resources, particularly firemen and ambulances. At that time the traffic and communication in the area were collapsed, therefore the dispatched resources (many of them can be considered HBS) had several problems to arrive to the emergency place, find the place assigned to park the emergency vehicles (depending on the role played by each resource type), find the incident commander (i.e., a HBS) and the command post, and get basic information about the emergency.

In the response process participated two helicopters, 110 ambulances, 55 police vehicles (over a hundred policemen) and 6 firefighting companies (over three hundred firemen). Fifteen hospitals received the injured people (a total of 676 people according to the official report [5]). The ambulances started transporting these people to the three closest hospitals. Once these hospitals were overcrowded, other options were taken. However, being this situation visible for the ambulances was a complex task that required a considerable time. Therefore, many ambulances tried to leave injured people in more than one hospital until find one able to receive them. The first paramedic (i.e. HBS) arriving to a collapsed hospital could record the hospital status in one or most witness units, and thus other ambulances accessing such an information can know where exactly to transport the people.

After the first 12 hours, no lists of dead and injured people were available. Therefore, relatives and friends of potential victims were asking in different hospitals and at the city morgue for hours, interfering with an already complex response process. The hospital managers (i.e. HBS) could have made public the list of injured people located there, e.g. through a Web portal or in a witness unit, allowing thus that civilians (also HBS) perform their searches quickly and without jeopardizing the response activities.

The search and rescue process took all day, and at the end there was still a missing person, who was found 48 hours later. He was a young man who got into the train 20 minutes before the crash and that was travelling in a compartment were passengers are not allowed to be. The rescuers went over the spot without noticing his presence.

This situation could be overcame due the public transportation system uses a contactless smart card, called SUBE (that stands for Electronic Ticket Unified System, in Spanish), which identifies each user. Making a small extension to the current infrastructure it is possible to know the number of people in the train, and also the identity 
and location of each passenger. If this information is shared through a witness unit, then it is possible to accelerate the response process, triggering parallel procedures that help reduce the damage. It also would have allowed an early detection of the missing person. An extra support for the people location could also be obtained using localization mechanisms based on cellular phones (with the WAP-OTA protocol).

Concerning communication support in the affected area, there is not clear information in the emergency reports, except that the telephone networks (wired and wireless) collapsed immediately after the incident and that were down for various hours. There is not report of the use of mobile antennas and only VHF radio systems seems to be used to support communication in the field. This situation is aligned with many others large incidents affecting urban areas [9], [11], [15].

The use of sensor networks, for example focused on traffic control, could have helped to change the frequency of the traffic lights when an emergency vehicle is approaching, or route the regular vehicles toward safe areas, where they do not interfere with the response process. Moreover, oppnet-based applications could be used to coordinate the response activities in the field and share valuable information to make local decisions. Although the technology to implement IoT-based solutions that support urban emergency responses is already available, there are no clear guidelines about the role played by each component type, how to integrate them, and how to design the behavior of an integral solution. In that sense, we hope that the proposed architecture and components characterizations contribute to reach such a goal.

\section{$5 \quad$ Conclusions and Future Work}

Urban incidents happen frequently in large cities and affect many people. Providing a fast an effective response process is critical to save lives and reduce the impact of these incidents on the civil property. This paper presents an architecture that helps design and implement IoT-based solutions that support first responses in urban areas. The proposal is particularly focused on large response processes, where many information producers and consumers need to be linked, and the information flow should be maximized. The architecture considers heterogeneous participants and communication networks. The example presented in Section 4 allows us to show that IoT-based solutions could play a key role in the improvement of responses to urban incidents.

Provided the role, behavior and services of the components considered in the architecture are clearly delimited, they can be modeled and formalized using a computable language. Thus, several IoT-based solutions adhering to the proposed architecture can be modeled, simulated and evaluated from a theoretical point of view, and then implemented when the designers are sure about the solution capabilities and limitations.

The use of a formal model for integrating the proposed components would also help evaluate vulnerability of urban areas and design coordination protocols between emergency response agencies. The next steps in this initiative are particularly focused on formalizing the architecture components and their interactions using a modeling computable language.

Acknowledgments. This work has been supported by Fondecyt (Chile), Grant $\mathrm{N}^{\circ}$ 1120207. 


\section{References}

1. Aldunate, R., Ochoa, S.F., Pena-Mora, F., Nussbaum, M.: Robust Mobile Ad-hoc Space for Collaboration to Support Disaster Relief Efforts Involving Critical Physical Infrastructure. ASCE Journal of Computing in Civil Engineering 20(1), 13-27 (2006)

2. Braunstein, B., Trimble, T., Mishra, R., Manoj, B., Lenert, L., Rao, R.: Challenges in Using Distributed Wireless Mesh Networks in Emergency Response. In: Proc. of ISCRAM 2006, NJ, USA, May 13-17 (2006)

3. Evanco, W.: The impact of rapid incident detection on freeway accident fatalities. Technical Report, Center for Information Systems, McLean,Virginia, USA (1996)

4. Feki, M.A., Kawsar, F., Boussard, M., Trappeniers, L.: The Internet of Things: The Next Technological Revolution. IEEE Computer 46(2), 24-25 (2013)

5. Government of the Buenos Aires city. Official Report of the Train Accident in Sarmiento Line,

http: / /buenosaires.gob.ar/2012-02-22-accidente-trensarmiento (last visit: September 15, 2013) (in Spanish)

6. IoT European Research Cluster. Internet of Things Strategic Research Roadmap. Cluster SRA (2011)

7. Lilien, L., Kamal, Z., Bhuse, V., Gupta, A.: Opportunistic networks: the concept and research challenges in privacy and security. In: Proc. of the Int. Workshop on Research Challenges in Security and Privacy for Mobile and Wireless Networks, Miami, USA (2006)

8. Liu, J.W.S., Chi-Sheng, S., Chu, E.T.-H.: Cyberphysical Elements of Disaster-Prepared Smart Environments. IEEE Computer 46(2), 69-75 (2013)

9. Martin-Campillo, A., Martí, R., Yoneki, E., Crowcroft, J.: Electronic triage tag and opportunistic networks in disasters. In: Proc. of the Special Workshop on Internet and Disasters, pp. 6:1-6:10. ACM Press, New York (2011)

10. Mendonça, D.: Decision Support for Improvisation in Response to Extreme Events: Learning from the Response to the 2001 World Trade Center Attack. In: Decision Support Systems, vol. 43(3), pp. 952-967 (2007)

11. Monares, A., Ochoa, S.F., Pino, J.A., Herskovic, V., Rodriguez-Covili, J., Neyem, A.: Mobile Computing in Urban Emergency Situations: Improving the Support to Firefighters in the Field. Expert Systems with Applications 38(2), 1255-1267 (2011)

12. Monares, A., Ochoa, S.F., Pino, J.A., Herskovic, V.: Improving the Initial Response Process in Urban Emergencies. In: Proc. of the IEEE CSCWD 2012, Wuhan, China, May 23-25 (2012)

13. Ochoa, S.F., Santos, R.: Human-centric Wireless Sensor Networks to Improve Information Availability During Urban Search and Rescue Activities. Inf. Fusion (in Press) (to appear)

14. Ozbay, K., Xiao, W., Jaiswal, G., Bartin, B., Kachroo, P., Baykal-Gursoy, M.: Evaluation of incident management strategies and technologies using an integrated traffic/incident management simulation. World Rev. of Int. Transp. Research 2(2/3), 155-186 (2009)

15. Panitzek, K., Schweizer, I., Bönning, T., Seipel, G., Mühlhäuser, M.: First responder communication in urban environments. Int. J. Mob. Netw. Des. Innov. 4(2), 109-118 (2012)

16. White, J., Thompson, C., Turner, H., Dougherty, B., Schmidt, D.C.: Wreckwatch: Automatic traffic accident detection and notification with smartphones. Mobile Network Applications 16(3), 285-303 (2011)

17. Yang, L., Yang, S., Plotnick, L.: How the internet of things technology enhances emergency response operations. Technol. Forecasting and Social Change (August 2012)

18. Zhang, J., Qi, A.: The application of Internet of things (IoT) in emergency management system in China. In: Proc. of the IEEE Conf. on Technol. for Homeland Security, November 8-10, pp. 139-142 (2010) 